\title{
Engagement des étudiants en tourisme, hôtellerie et restauration envers leur domaine d'études et leur carrière : les facteurs déterminants
}

Commitment of tourism, hotel and restaurant management students to their field of study and their career: determining factors

Jean Lagueux et Audrey Nanot

\section{OpenEdition}

\section{Journals}

Édition électronique

URL : http://journals.openedition.org/tourisme/2414

DOI : 10.4000/tourisme.2414

ISSN : 2492-7503

Éditeur

Éditions touristiques européennes

Référence électronique

Jean Lagueux et Audrey Nanot, « Engagement des étudiants en tourisme, hôtellerie et restauration envers leur domaine d'études et leur carrière : les facteurs déterminants », Mondes du Tourisme [En ligne], 16 | 2019, mis en ligne le 01 décembre 2019, consulté le 09 avril 2020. URL : http:// journals.openedition.org/tourisme/2414; DOI : https://doi.org/10.4000/tourisme.2414

Ce document a été généré automatiquement le 9 avril 2020.

Mondes du tourisme est mis à disposition selon les termes de la licence Creative Commons Attribution - Pas d'Utilisation Commerciale - Pas de Modification 4.0 International. 


\section{Engagement des étudiants en tourisme, hôtellerie et restauration envers leur domaine d'études et leur carrière : les facteurs déterminants}

Commitment of tourism, hotel and restaurant management students to their field of study and their career: determining factors

Jean Lagueux et Audrey Nanot

\section{Introduction}

1 D'ici 2035, la croissance de l'industrie touristique canadienne risque d'être sérieusement compromise du fait d'une pénurie annoncée de 235000 travailleurs (Conference Board Canada, 2016), ce qui amène les établissements d'enseignement en tourisme, hôtellerie et restauration (THR) à se questionner sur les moyens de recrutement et de formation à mettre en œuvre pour attirer des candidats ayant un fort potentiel d'engagement envers leur future carrière.

2 En effet, si c'est souvent la passion d'un métier qui amène un individu à choisir une carrière, la réalité du milieu professionnel peut parfois apparaître comme étant en porte-à-faux par rapport aux aspirations profondes de ces mêmes individus. Afin de répondre à cette problématique, la présente recherche identifie des facteurs ayant une influence sur le degré d'engagement d'un étudiant à persévérer dans le domaine choisi. Ces facteurs peuvent être nombreux, depuis les conditions socioéconomiques d'un territoire, en passant par la culture d'une industrie, le type d'emploi jusqu'au profil de l'individu. Cependant, cette recherche écartera l'analyse des milieux de travail pour 
mieux saisir le ressenti de l'individu par rapport à cet environnement, en lien avec les expériences professionnelles vécues avant et au cours de son parcours académique.

\section{La revue de littérature}

3 Alors que de nombreuses recherches en gestion ont identifié les facteurs favorisant la loyauté des individus à une organisation, peu ont lié les traits intrinsèques d'un étudiant, en processus d'apprentissage, à sa volonté de persévérer dans le domaine choisi (Costen et Salazar, 2011). Afin d'aborder cette problématique, les concepts entourant la carrière d'un individu, l'appréciation de son environnement de travail et les intentions reliées au domaine d'études choisi ont, dans un premier temps, été extraits de la littérature existante sur le sujet. Dans un deuxième temps, le concept d'expérience émotionnelle en milieu de travail, qui fait état du ressenti de l'individu lorsqu'il est en milieu de travail, est défini à partir des écrits existants. Enfin, à partir des recherches effectuées sur les employés de services en THR, certaines prédispositions de l'individu sont précisées.

\section{Engagement envers sa carrière}

4 Premièrement, au cœur de la problématique de cette recherche, se situe la propension d'un employé à s'engager dans différents cadres organisationnels et certaines industries. Au-delà de la loyauté envers son organisation, le construit d'engagement envers sa carrière est ici plus pertinent (Darden et al., 1989).

5 Les travaux de London et Noe (1997) font état d'un ensemble de facteurs endogènes et exogènes à l'individu pouvant être reliés à sa motivation à poursuivre ou non dans la même voie de carrière. La revue de littérature qui suit relève un ensemble de paramètres évoqués dans la littérature scientifique de la gestion des ressources humaines et du comportement organisationnel afin de construire un modèle d'analyse pouvant expliquer la motivation sous-tendant la loyauté envers sa carrière.

6 Afin de mieux cerner cette propension de l'individu à s'engager, les travaux de Carson et Bedeian (1994) arrivent à un construit composé de trois dimensions. La première examine comment les individus se projettent dans les tâches à accomplir et comment ils s'identifient ultimement à leurs responsabilités professionnelles. La deuxième dimension du concept, la planification de carrière, fait référence au contrôle exercé par les individus sur les mesures conscientes qu'ils ont prises pour planifier leur carrière, c'est-à-dire leur capacité à faire des choix qui font partie d'un schéma interne et qui sont alignés à leurs convictions personnelles ou à leurs croyances professionnelles. La troisième et dernière dimension, la résilience professionnelle, examine la capacité des employés à travailler avec un certain degré de pression, à réagir de manière professionnelle aux défis imprévus et à conserver l'énergie nécessaire pour continuer sur la voie professionnelle librement choisie (Darden et al., 1989).

7 Dans la même veine, la recherche sur la persévérance scolaire est prolifique et a hautement contribué à mieux comprendre la progression des étudiants dans leur cheminement scolaire. Le fait d'avoir un emploi rémunéré pendant ses études est désormais reconnu comme ayant des effets négatifs sur la continuité des études (Maurel et al., 2009). Toutefois, la littérature s'est peu attardée à saisir les effets de la poursuite d'un programme d'études sur la continuité du choix de carrière d'un 
étudiant. Ainsi, l'intention de terminer son programme d'études, voire de changer de programme ou de travailler dans un autre champ d'activité n'est pas documentée. Cette étude s'intéresse donc à la continuité de carrière sous le regard de la persévérance scolaire.

\section{L'appréciation de l'expérience au travail}

$8 \quad$ Afin de mieux saisir les facteurs agissant sur la capacité à se projeter dans le futur, les construits issus des mesures de performance au travail peuvent être considérés. Ainsi, la littérature en gestion des ressources humaines continue de lier le sentiment positif ressenti par un individu en milieu de travail à sa performance et à sa volonté de persévérer. Partant de ce consensus, la dimension propre à la satisfaction au travail demeure à ce jour un construit incontournable dans l'étude des liens que les employés peuvent entretenir avec leur emploi (Brown et Lam, 2008).

Dans le but d'approfondir cette appréciation du milieu de travail, de nouveaux construits émergent afin de saisir d'autres aspects de la dynamique de travail de l'employé. Ainsi, le concept d'expérience au travail apparaît au tournant des années 1970 et a depuis intégré un certain nombre de perspectives liées aux notions de qualité, de valeur et d'émotions. En effet, dans le but de réduire les taux de rotation de leur personnel, les organisations ont commencé à inclure à leurs pratiques de gestion des ressources humaines des approches de marketing interne afin de résoudre les problèmes liés à l'engagement des employés (Jones, 2008). Or, le concept d'expérience dans un milieu professionnel a rapidement pris une perspective différente que celle utilisée pour mesurer la satisfaction des clients. Des concepts tels que le travail émotionnel des employés (Lee et Ok, 2012; Seymour, 2000), le bien-être individuel (Johnson et Spector, 2007), le plaisir et la satisfaction au travail (Young Gin et al., 2013) ont permis de mettre en lumière de nouveaux aspects propres à la compréhension de la performance émotionnelle des employés de service. Alors que ce dernier concept, la satisfaction en milieu de travail, s'avère être une mesure a posteriori d'un événement, plusieurs chercheurs ont tenté de saisir la validité de l'expérience émotionnelle propre au moment de l'épisode de travail. Dans cette optique, le degré de plaisir et d'excitation ressenti par l'employé devient un facteur de plus en plus utilisé pour caractériser l'environnement de travail (Plester et Hutchison, 2016). Cette perspective, axée sur l'impact à moyen terme de l'expérience professionnelle vécue par le prestataire, sera donc celle priorisée dans le cadre de cette étude.

\section{Les prédispositions des individus}

10 Comme mentionné par London et Noe (1997), un regard sur le travailleur - l'étudiant dans le cadre de notre étude - et de ses prédispositions s'avère judicieux afin d'orienter une lecture juste du profil d'individus les plus enclins à se démarquer dans ce type d'environnement et à se projeter dans leur choix de carrière.

La littérature propre au milieu THR a largement étudié les caractéristiques individuelles qui correspondent le mieux aux emplois liés à la prestation de services. Ainsi, la qualité du service offert et la performance globale des agents ont souvent été examinées à travers l'étude des traits de personnalité (Lee-Ross et Pryce, 2005), des 
prédispositions innées (Winsted, 1997), des compétences acquises, des valeurs personnelles et des éléments culturels (Johns et al., 2007).

\section{Les antécédents culturels}

12 L'une des tâches essentielles de tout employé en THR est d'accueillir et de servir des invités de toutes origines culturelles et ethniques. Par conséquent, il existe une abondante littérature explorant l'empreinte culturelle individuelle comme antécédent à la performance professionnelle (Lee et al., 2018 ; Taylor et McArthur, 2009). De plus, les marqueurs culturels sont plus que jamais des repères significatifs lorsqu'on tente d'expliquer la relation que certains individus entretiennent avec leur milieu de travail (Sizoo et al., 2004). Lorsque l'on considère les caractéristiques propres à l'expression culturelle dans des cadres conceptuels, les construits issus des recherches de Geert Hofstede sont souvent les premiers utilisés (Yoo et al., 2011). Parmi les six dimensions examinées pour mesurer l'impact de la culture sur l'organisation du travail, l'orientation individualisme/collectivisme et le degré de distance hiérarchique perçu dans les relations professionnelles sont les deux dimensions les plus couramment utilisées pour explorer le sentiment d'identité ou d'appartenance des employés (Hofstede et al., 2017). Mentionnons que les recherches d'Hofstede sont orientées vers la mesure de traits caractéristiques à toute une nation (Bearden et al., 2006). Or, si l'on désire se rapprocher des prédispositions individuelles, une autre perspective sur la culture doit être adoptée. Ainsi, une dimension qui reprend de l'importance dans les milieux de travail du THR est la présence d'environnements plus ou moins formels, dans lesquels il existe une distance hiérarchique plus ou moins forte entre les individus (Dash et al., 2006). De plus, ces milieux de travail sont également caractérisés par la présence d'individus dont l'expression du comportement varie d'une approche plus individualiste ou collectiviste (Johns et al., 2007). Ces deux dimensions à elles seules permettent de situer plus précisément l'individu quant à sa capacité à lire les attentes inhérentes à son comportement au sein d'une équipe de travail, mais également à y répondre adéquatement dans une structure plus ou moins statique quant à son organisation hiérarchique. Différentes études dans le milieu THR (Koc, 2013; White, 2005) ont en outre démontré le lien positif entre la perception de la distance hiérarchique, d'une organisation de travail plus collectiviste qu'individualiste et le degré de satisfaction ressentie par l'employé de service; ces deux dimensions apparaissent donc comme des indicateurs fiables à considérer dans l'explication des facteurs liés à l'appréciation du milieu de travail.

\section{L'orientation client des employés de services}

13 La nature des tâches des emplois en THR est par ailleurs spécifique au domaine des services, dans un environnement de consommation hédonique, et requiert de faire le lien entre le profil des employés de service et la satisfaction qu'ils peuvent apporter à la clientèle lors des interactions de service. Le concept de COSE (Customer Oriented Service Employee, ou employé de service orienté client) (Kang et Hyun, 2012), défini comme étant la tendance ou la prédisposition d'un prestataire de service à répondre adéquatement aux besoins d'un client dans un contexte professionnel (Brown et al., 2002), s'avère judicieux pour analyser l'engagement du travailleur dans ce contexte de travail particulier. Ce construit est composé de quatre dimensions qui deviennent de 
bons indicateurs pouvant influencer l'appréciation du milieu de travail (Kang et Hyun, 2012). La première dimension fait référence au niveau de compétences techniques acquis par les employés, les rendant plus ou moins bien outillés pour répondre aux besoins des clients lorsqu'en situation de prestation de service. La deuxième dimension, axée sur les compétences sociales, analyse la capacité des employés à dialoguer de manière significative avec les clients afin de bien percevoir ce que les clients communiquent et ce qu'ils ressentent. La troisième dimension aborde le degré de motivation : elle fait référence au degré d'engagement des employés à l'égard de leur travail, tel que démontré dans leurs interactions avec les clients. Enfin, la dimension de l'autorité décisionnelle fait référence à la capacité des employés à prendre des décisions de manière autonome sans avoir à vérifier avec les supérieurs hiérarchiques. Ce concept se distingue de celui de l'autonomisation, qui est l'autorité donnée par l'organisation aux employés afin qu'ils prennent leurs propres décisions. Cette prédisposition envers le client s'est révélée être un indicateur fiable permettant de relier le concept de COSE à la satisfaction du client ou à la qualité du service (HennigThurau, 2004).

\section{Le locus de contrôle}

Enfin, et dans le but de préciser le profil d'employé souhaité, d'autres dimensions de l'individu présentent un intérêt pour saisir le degré d'engagement de l'employé. Notamment, le concept de "locus de contrôle", qui a largement été utilisé dans les domaines du leadership et de la motivation, permet de mesurer la faculté de l'individu à percevoir l'inhérence des événements de son existence comme étant propre à sa volonté ou externe à ses décisions (Rotter, 1966). Dans les faits, les individus qui estiment avoir un certain contrôle sur leur destin - locus de contrôle interne - ont tendance à démontrer une plus grande compréhension de leur lieu de travail que ceux qui pensent que le monde extérieur contrôle leur destin (Salazar et al., 2002). De plus, dans le milieu THR, Silva (2006) a, plus spécifiquement, constaté que les attitudes des employés vis-à-vis de leur emploi, en particulier leur engagement envers l'organisation et leur satisfaction au travail, étaient étroitement liées à la mesure de leur locus de contrôle. Cette dimension présente donc un fort intérêt lorsque pour comprendre l'adéquation entre l'individu et la nature de l'emploi.

\section{Le model conceptuel}

Le modèle conceptuel émanant de cette revue de littérature est donc fondé sur les travaux de London et Noe (1997) et sur la théorie de motivation de carrière. London et Noe (1997) se réfèrent à un certain nombre de facteurs situationnels qui, eux-mêmes, émanent des théories de la motivation. Cette théorie fait ressortir les facteurs de motivation reliés au développement de carrière chez les individus et tente d'isoler certains de ces facteurs, endogènes ou exogènes, ayant des liens avec la progression professionnelle.

16 Ainsi, un modèle conceptuel dérivé de la littérature sur le comportement organisationnel et les ressources humaines relie un certain nombre de prédispositions des employés en tentant compte d'une nouvelle dimension, à savoir le degré de plaisir vécu lors d'un épisode de travail. 

individuelles à une variable d'appréciation du milieu de travail, elle-même reliée au concept d'engagement de carrière et à trois dimensions d'intentions futures des participants.

18 L'hypothèse $\mathrm{n}^{\circ} 1$ examine la relation entre l'appréciation en milieu de travail (expérience émotionnelle) et l'engagement professionnel, tandis que l'hypothèse $n^{\circ} 2$ énonce un certain nombre de relations entre cette même appréciation en milieu de travail et les intentions futures des étudiants. Enfin, l'hypothèse $n^{\circ} 3$ énonce les relations attendues entre des facteurs endogènes à l'individu et l'appréciation de l'environnement de travail. La figure 1 illustre l'ensemble d'hypothèses entre chaque construit. Le tableau 1 énonce les relations attendues et présente les résultats obtenus.

Figure 1. Cadre conceptuel

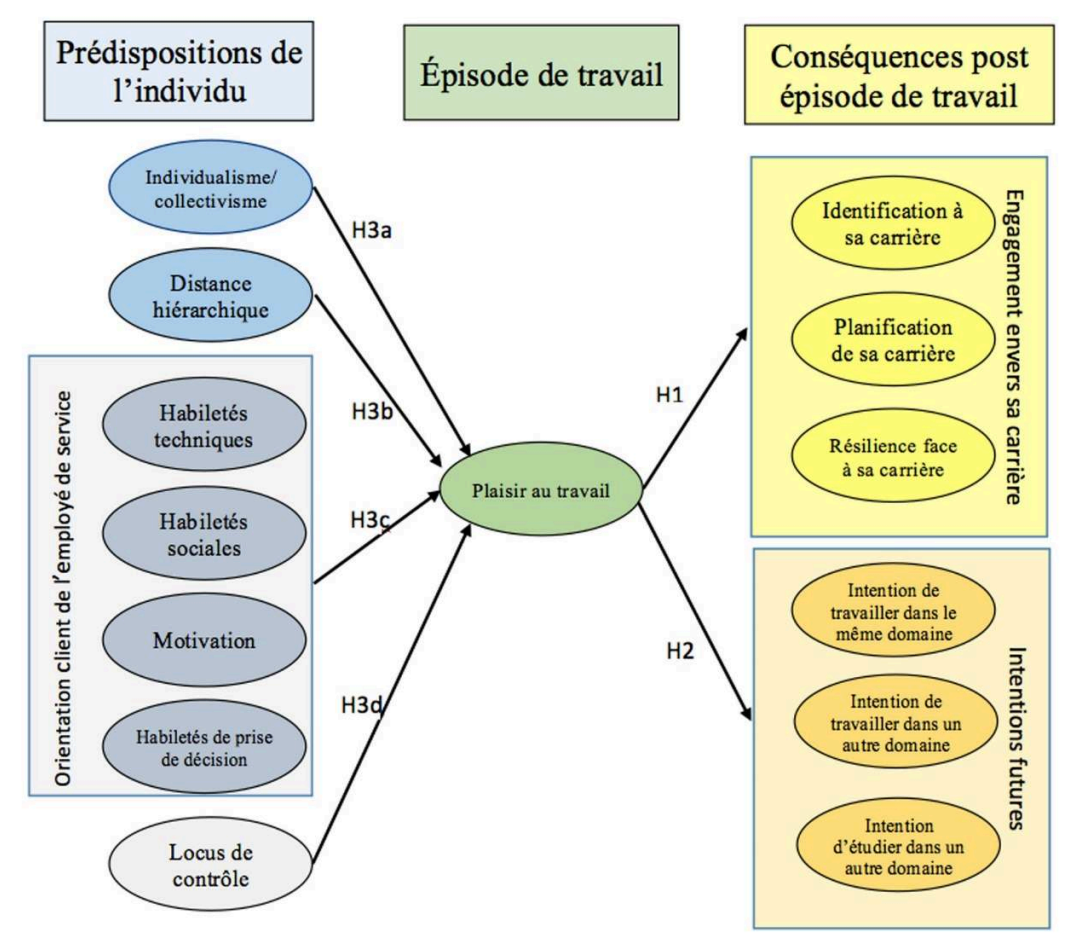

\section{La méthodologie}

Afin de mener à bien cette recherche, un échantillon de 600 étudiants, fréquentant tous le même établissement, a été sollicité. Cet établissement propose trois ordres distincts d'enseignement, dont deux ont été sondés : l'ordre collégial (trois techniques distinctes en tourisme, hôtellerie et restauration) ${ }^{1}$ et universitaire (en tourisme et hôtellerie). Sur ce nombre de possibles répondants, 465 questionnaires ont été recueillis, dont 424 ont finalement été conservés pour la validité de leurs résultats. Le questionnaire, développé en utilisant des échelles de mesure déjà existantes, a été distribué en classe.

L'analyse factorielle confirmatoire n'a pas confirmé la présence d'une structure factorielle de second ordre pour les construits de COSE et d'engagement de carrière. Les 
facteurs individuels ont été traités séparément dans le cadre de l'analyse. De plus, afin d'obtenir une cohérence interne, le coefficient alpha de Cronbach a été évalué pour toutes les échelles (Nunnally, 1978).

\section{Le profil de l'échantillon}

$21 \mathrm{Au}$ total, parmi notre échantillon de 424 répondants, $66 \%$ des individus se sont identifiés comme étant des femmes et $44 \%$ comme étant des hommes. $42 \%$ des participants étaient en première année d'étude, $30 \%$ en deuxième année et $28 \%$ en troisième année ou plus. En ce qui concerne les groupes d'âge, $45 \%$ de l'échantillon avaient entre 17 et 20 ans, $45 \%$ entre 21 et 25 ans et $10 \% 26$ ans et plus. La répartition des étudiants par programme d'études a révélé que $33 \%$ des étudiants suivaient un programme international de gestion hôtelière, $23 \%$ étaient dans un programme de gestion de la restauration, $23 \%$ dans un programme de gestion touristique (trois niveaux collégiaux) et $21 \%$ faisaient partie d'un programme de gestion touristique et hôtelière au niveau universitaire.

S'agissant de l'expérience de travail, $27 \%$ des participants avaient de 0 à 6 mois d'expérience de travail dans leur domaine d'études, $19 \%$ avaient de 7 à 12 mois d'expérience, $18 \%$ avaient de 13 à 24 mois d'expérience, $18 \%$ avaient 48 mois d'expérience et $18 \%$ avaient 49 mois ou plus d'expérience professionnelle.

\section{Le portrait des répondants}

23 Afin d'obtenir une meilleure compréhension de notre échantillon de population en fonction de leurs caractéristiques individuelles perçues et liées aux variables choisies, un portrait partiel a été dressé.

Dans l'ensemble, les étudiants s'estiment plus collectivistes qu'individualistes $7=5,2 / 7)$ et ils manifestent aussi une appréhension de la distance hiérarchique plus faible $(=2,3 / 7)$, ce qui démontre un sens plus élevé de l'égalité en termes de perception de la distribution du pouvoir dans leur environnement. Quant à l'expression de leur orientation client, ils ont déclaré posséder de bonnes compétences techniques $7=5.6 / 7)$ et d'excellentes aptitudes sociales $7=6.3 / 7)$, qui seraient typiques des étudiants formés en programmes d'accueil. La troisième dimension du COSE, la motivation perçue, a obtenu une moyenne de $6,2 / 7$, ce qui illustre une plus grande perception de leur propre désir de faire les efforts appropriés pour accomplir une tâche professionnelle donnée.

Pour la dernière prédisposition étudiante, les résultats montrent qu'ils se perçoivent comme ayant un locus de contrôle plus interne qu'externe $(=5,5 / 7)$, ce qui indique qu'ils sont dotés de la croyance personnelle d'avoir le contrôle de leur propre vie. Les résultats liés aux épisodes de travail vécus par les étudiants révèlent par ailleurs qu'ils ont surtout ressenti un sentiment de plaisir au travail ( = 5,4 / 7).

Enfin, en termes d'engagement dans leur carrière, les résultats montrent que les étudiants ont tendance à s'identifier très fortement à leur carrière $7=6,0 / 7)$ mais qu'ils sont moins enclins à se projeter dans un plan de carrière $(=4,9 / 7)$ et qu'ils étaient également moins résilients donc moins outillés pour rebondir face aux défis liés au travail $(=4.4 / 7)$. 


\section{Les résultats}

Des analyses de régression simple et multiple ont été utilisées pour étudier le lien entre ce que les étudiants ont vécu, expérimenté au cours de leur épisode de travail et les trois dimensions de l'engagement professionnel. Ces trois dernières montrent certaines relations. Le tableau 1 et la figure 2 rapportent les résultats au regard des hypothèses qui ont été confirmées.

Figure 2. Résultats des hypothèses de recherche

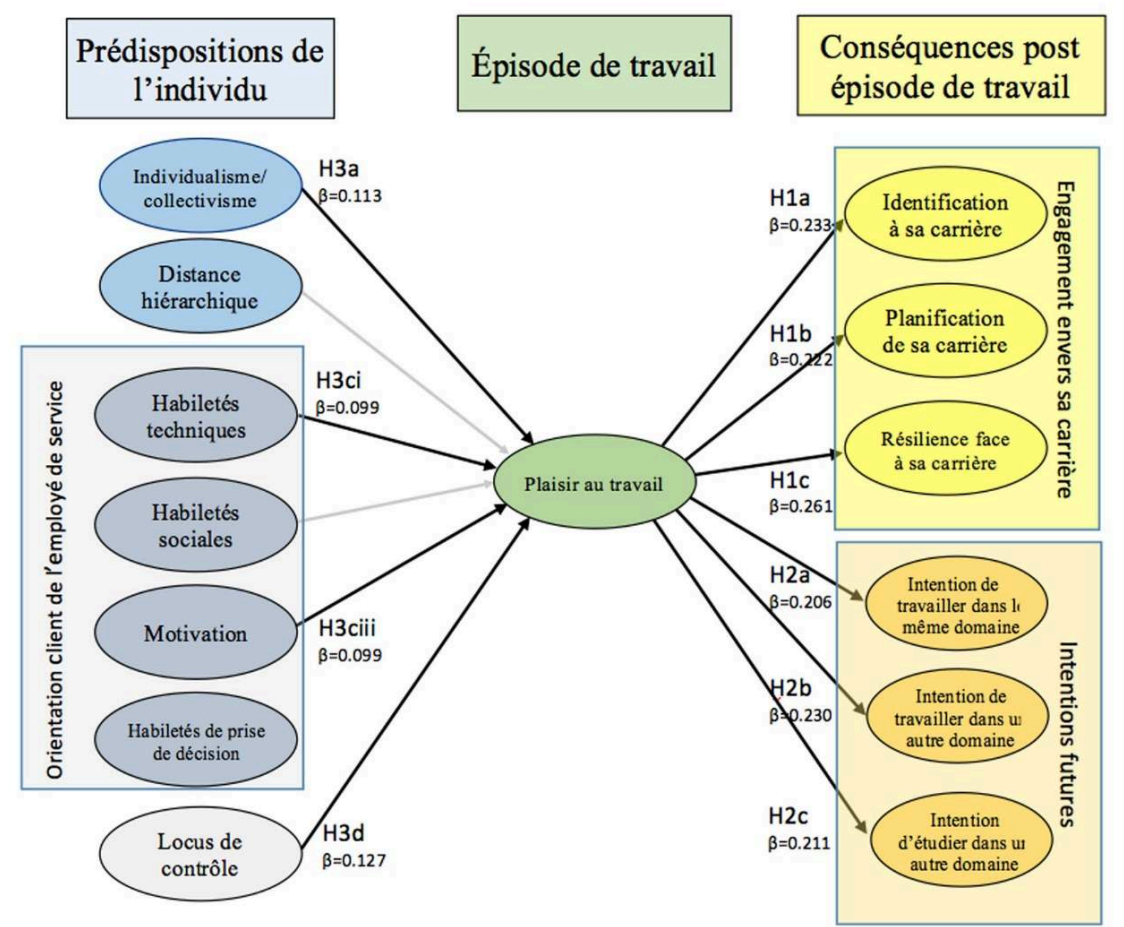


Tableau 1. Synthèse des hypothèses et des résultats
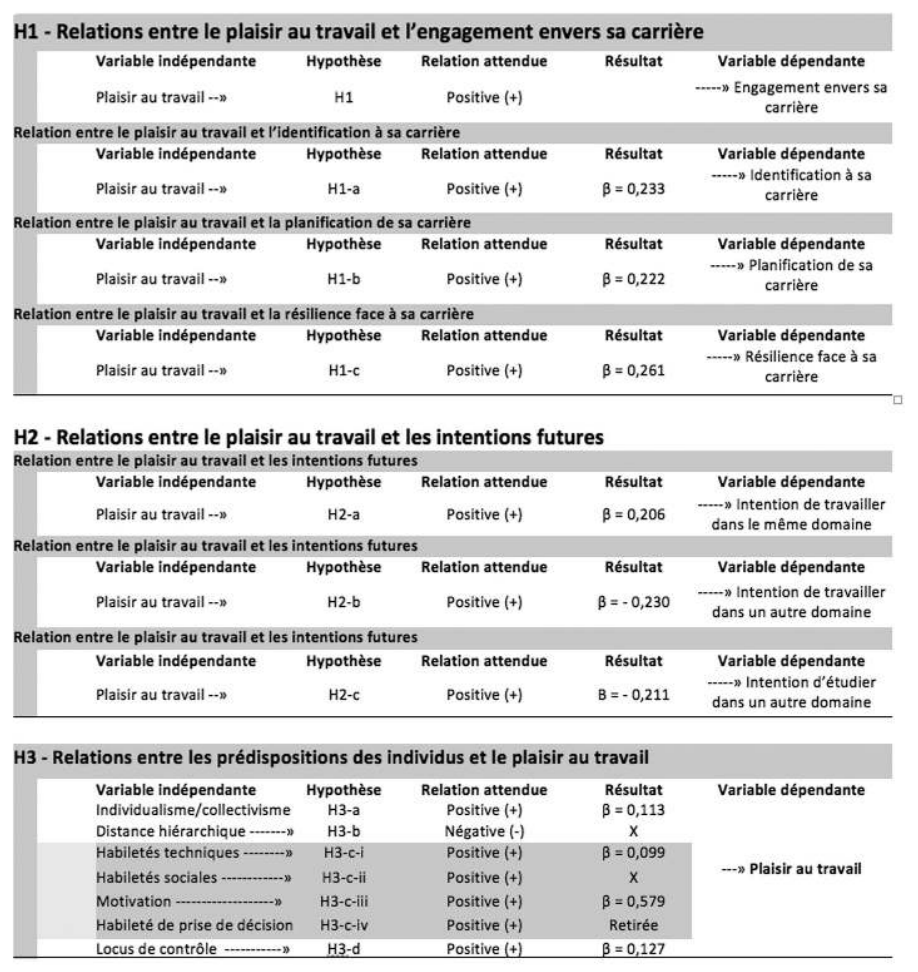

La première série d'analyses examine la relation entre les épisodes de travail des étudiants et l'engagement envers leur carrière. Premièrement, une analyse de régression simple a été utilisée avec l'identification de la carrière comme critère et les variables associées aux épisodes de travail des étudiants comme prédicteurs. Les résultats de l'analyse de régression indiquent qu'il existe une relation positive entre le degré d'expérience émotionnelle vécue par les répondants (H1a: $\beta=0,233$, Rdeux $=0,026$ ) et la façon dont ils s'identifient à leur carrière, ce qui signifie que plus le travail est agréable, plus ils se sentent connectés à la profession choisie. Pour ce qui est de la seconde dimension, l'engagement envers sa carrière ou la planification, un lien positif est également obtenu $(\mathrm{H} 1 \mathrm{~b}: \beta=0,222$, Rdeux $=0,026)$. Enfin, pour la troisième dimension, l'appréciation de l'expérience en milieu de travail montre un lien positif avec la résilience face à sa carrière ( $\mathrm{H} 1 \mathrm{c}: \beta=0,261$, Rdeux $=0,029)$. La variance expliquée par ces trois facteurs est très basse, ce qui indique que bien d'autres facteurs peuvent en effet expliquer la variabilité associée à l'appréciation de l'expérience. Ces résultats indiquent qu'une personne ayant une expérience émotionnelle positive sera plus disposée à s'identifier à sa carrière, à la planifier et sera plus résiliente à travers sa carrière.

Ces résultats sont conformes à d'autres études, dans lesquelles le facteur du plaisir (les émotions positives au travail) est relié à des concepts similaires liés à l'emploi, tels que l'intégration au travail (Tews et Michel, 2015) et l'engagement envers ses tâches (Plester et Hutchison, 2016). Ils convergent également avec les travaux de Kong et Jiang (2018) qui précisent que la présence d'émotions positives au travail, au travers de la présence d'une intelligence émotionnelle supérieure, ont un lien positif avec l'établissement d'objectifs reliés à sa carrière. Par conséquent, les présents résultats confirment la pertinence du concept de plaisir au travail dans des modèles expliquant 
l'engagement des employés, au-delà de leur emploi, envers leur carrière. Cette relation entre le plaisir vécu au travail et l'engagement envers sa carrière nécessitera d'être investiguée davantage.

Le deuxième niveau d'analyse porte sur les liens entre l'expérience émotionnelle des étudiants et leur intention de continuer à étudier ou travailler dans leur domaine de carrière ou dans un autre domaine. Les résultats montrent qu'il existe un lien entre l'expérience émotionnelle du participant, en milieu de travail, et la suite de ses études. Ainsi, plus l'expérience émotionnelle est positive, plus l'individu a l'intention de travailler dans le même domaine (H2a $: \beta=0,206$, Rdeux $=0,002$ ). Ce résultat est aussi en lien avec la relation négative qui existe entre l'expérience émotive positive et le désir de vouloir travailler dans un autre domaine $(\mathrm{H} 2 \mathrm{~b}: \beta=-0,230$, Rdeux $=0,018)$. D'autre part, il existe aussi un lien négatif significatif entre l'expérience émotionnelle en milieu professionnel et l'intention de poursuivre ses études dans un autre domaine $(\mathrm{H} 2 \mathrm{c}: \beta=-$ 0,211 , Rdeux $=0,130$ ), ce qui signifie que plus l'expérience émotionnelle est positive, moins l'étudiant sera enclin à étudier dans un autre domaine.

Bien que le modèle de Tinto (Bers et Smith, 1991) soit la référence en termes de persévérance scolaire, peu d'études ont été menées pour examiner le lien entre le désir de l'étudiant de poursuivre ses études dans un même domaine et les expériences professionnelles vécues dans ce même domaine d'activité. Le modèle de Tinto comprend deux dimensions, garantes de persévérance: l'intégration académique et l'intégration sociale. Les recherches effectuées sur la relation entre ces deux concepts et les intentions d'études demeurent mitigées et présentent souvent des résultats contradictoires (Bers et Smith, 1991). L'intégration professionnelle, à adapter aux programmes en alternance travail-études, serait alors une nouvelle dimension à considérer dans la persévérance scolaire.

Le troisième niveau d'analyse porte sur les liens entre les prédispositions des étudiants et leur appréciation de leur épisode de travail en tant qu'expérience émotionnelle. Une régression multiple, avec l'appréciation de l'expérience de travail comme variable dépendante, montre que les variables explicatives du modèle expliquent $21,2 \%$ de la variance.

Les résultats de cette régression multiple montrent, dans un premier temps, qu'il existe un lien positif entre le fait de se percevoir comme plus collectiviste qu'individualiste (H3a : $\beta=0,113)$ et le fait de vivre des émotions plus positives sur le lieu de travail. Ce résultat est soutenu par les recherches de Hui et al. (1995) qui ont constaté que «les employés collectivistes ont déclaré être plus satisfaits de leur travail, salaire, promotion, supervision et collègues que leurs homologues individualistes ». Ainsi, le trait collectiviste semble être moins lié aux émotions personnelles négatives. Les résultats actuels confirment donc que le collectivisme et l'individualisme demeurent des facteurs pertinents qui influent sur les résultats liés au travail.

Deuxièmement, également issu du concept de COSE, le fait de se percevoir comme possédant de bonnes compétences techniques a aussi un lien positif (H3c-i : $\beta=0,099)$ avec des émotions plus positives. Cet effet se traduit aussi au niveau de la motivation envers son travail (H3c-iii : $\beta=0,579$ ). Cela signifie que les individus éprouvent plus de plaisir au travail lorsqu'ils se sentent mieux outillés d'un point de vue technique et qu'ils sont plus motivés. Enfin, lorsque les étudiants démontrent un locus de contrôle plus interne, une relation positive significative $(\mathrm{H} 3 \mathrm{~d}: \beta=0,127)$ existe avec la présence d'émotions positives, ce qui signifie que les étudiants qui ont l'impression de mieux 
maitriser leur destin expérimentent davantage de plaisir au travail. Cette relation positive entre le locus de contrôle des étudiants et l'expérience émotionnelle confirme les résultats de Silva (2006) sur les attitudes professionnelles et l'engagement organisationnel, dans lesquels cinq grands traits de personnalité et le locus de contrôle sont positivement reliés à la satisfaction professionnelle des employés et leur engagement. Cela confirme également les résultats de nombreuses autres études : un locus de contrôle interne est positivement lié à des résultats de performance plus souhaitables (Dijkstra et al., 2011; Twenge et al., 2004). Les résultats de cette étude offrent donc une nouvelle perspective en confirmant que le locus de contrôle a aussi une relation positive sur les émotions positives expérimentées en milieu professionnel par les employés.

\section{Conclusion} individu porté à connaître des expériences positives en milieu professionnel et à ultimement persévérer dans l'industrie. L'adéquation entre la satisfaction, l'engagement et la persévérance est aussi probante. La richesse de ces résultats peut s'avérer utile pour les recruteurs académiques afin de cibler au mieux les étudiants les plus prompts à persévérer, mais permet aussi de détecter les individus dont la performance pourrait être accrue dans leur première année de travail si le milieu professionnel présente une forte adéquation avec la perception de plaisir et ultimement le souvenir de satisfaction.

Plus spécifiquement, les résultats de cette étude peuvent aider les directions d'établissement en milieu THR à développer l'offre d'emploi proposée aux étudiants au cours de leurs programmes d'études. Lorsque les établissements aident les étudiants à trouver un stage rémunéré, un emploi complémentaire à leur scolarité ou un poste estival, ils peuvent se référer à ces résultats pour faire le lien avec les profils des étudiants et pour ainsi mieux les préparer afin d'optimiser leur niveau d'engagement envers leur future carrière. Ainsi, le fait de mieux comprendre le rôle des expériences émotionnelles dans les stages professionnels peut les amener à se concentrer sur des organisations qui visent à créer de véritables expériences positives pour leurs employés et qui valorisent la notion de plaisir au travail. Puisqu'il existe un lien positif entre le facteur du plaisir au travail et l'avenir des carrières des étudiants, il est donc logique que les établissements d'enseignement et leurs représentants travaillent avec des organismes qui visent à améliorer le niveau de plaisir au travail afin de garder les étudiants dans le domaine de l'hospitalité. Les établissements d'enseignement formant les futurs gestionnaires et la main d'œuvre en milieu THR peuvent également travailler avec les étudiants afin de les aider à mieux appréhender l'importance du plaisir au travail et leur enseigner des façons d'allouer du temps pour valoriser ce facteur.

Éduquer les étudiants à apprécier leurs propres compétences techniques peut également mieux les préparer à leur environnement de travail. En effet, sensibiliser les étudiants au fait que plus ils se sentent compétents au travail sur le plan technique, plus ils sont susceptibles de vivre une expérience émotionnelle positive au travail, peut inciter les étudiants à se concentrer sur l'acquisition de ces compétences. Les enseignants dont le champ d'expertise touche plus spécifiquement l'accueil peuvent également se concentrer sur les impacts positifs de bonnes compétences sociales et

Mondes du Tourisme, 16 | 2019 
animer des ateliers et des sessions de formation dédiés afin d'outiller les étudiants pour qu'ils deviennent des communicateurs efficaces, dotés de compétences sociales exceptionnelles. De plus, il serait aussi tout à fait à propos de proposer des programmes qui misent plus particulièrement sur l'expérience affective positive en milieu de travail. Enfin, les établissements d'enseignement devraient prendre en compte le puissant concept de locus de contrôle lors du recrutement des étudiants. La sensibilisation à l'autodétermination et un locus de contrôle plus interne peuvent également permettre aux étudiants d'avoir une meilleure appréciation de leur lieu de travail, tout en s'amusant davantage. Sensibiliser les étudiants à prendre conscience de leur locus de contrôle interne par des activités de mentorat, de coaching ou de jeux de rôles peut être un moyen efficace de démontrer un comportement plus approprié au travail, de promouvoir les émotions positives au travail, qui s'avèrent in fine bénéfiques pour leur engagement envers leur carrière.

Finalement, il serait pertinent d'interpeller les responsables du recrutement dans les organisations touristiques misant sur certaines dimensions propres à la qualité du service à la clientèle et surtout à la rétention de leurs employés. En effet, ces résultats pourraient aussi être vulgarisés et communiqués auprès des recruteurs afin de valoriser le fait que les prédispositions les plus gagnantes chez les futurs candidats sont celles axées sur le locus de contrôle et sur la mise en lumière des habilités techniques et sociales.

\section{Limites de la présente étude}

Bien que les résultats de la présente étude se limitent à une seule population scolaire, ils révèlent des relations significatives entre des concepts qui n'avaient pas été reliés auparavant et ils fournissent des conseils utiles sur la façon d'améliorer les modèles théoriques futurs. Cela permettra d'enrichir la compréhension globale de l'engagement professionnel des étudiants pour la nouvelle génération de travailleurs plus diversifiée entrant sur le marché du travail.

41 Ces résultats appellent néanmoins des études sur d'autres populations scolaires, dans des contextes sociaux et culturels différents, afin de mieux comprendre les mécanismes de la gestion de carrière. De plus, des études portant sur d'autres prédispositions, telles que l'intelligence émotionnelle, l'empathie, l'auto-efficacité, l'adaptabilité, les compétences de communication, entre autres, permettraient certainement de comprendre comment l'engagement professionnel peut être façonné et renforcé.

Dernièrement, l'engagement professionnel devrait également être envisagé sous un angle différent en tenant compte du facteur de longévité, plus précisément en extrapolant le modèle actuel afin d'étudier les facteurs qui permettraient aux travailleurs plus âgés de poursuivre leur carrière plus longtemps.

L'engagement professionnel est complexe et multidimensionnel et l'industrie THR est aujourd'hui confrontée à des changements démographiques et à la nécessité de trouver de nouvelles façons d'offrir une expérience distincte à sa clientèle. Des recherches supplémentaires sont donc nécessaires pour affiner ces résultats. 


\section{BIBLIOGRAPHIE}

William O. Bearden, R. Bruce Money et Jennifer L. Nevins, « Multidimensional versus unidimensional measures in assessing national culture values: The Hofstede VSM 94 example », Journal of Business Research, 59(2), 2006 [http://dx.doi.org/10.1016/j.jbusres.2005.04.008].

Trudy H. Bers et Kerry E. Smith, « Persistence of community college students: The influence of student intent and academic and social integration », Research in Higher Education, 32(5), 1991 [doi : 10.1007/BF00992627].

Steven Brown et Son Lam, « A Meta-Analysis of Relationships Linking Employee Satisfaction to Customer Responses », Journal of Retailing, 84(3), 2008 [doi : 10.1016/j.jretai.2008.06.001].

Tom J. Brown, John C. Mowen, Todd Donavan et Jane W. Licata, « The customer orientation of service workers: Personality trait effects on self and supervisor performance ratings ", Journal of Marketing Research, 39(1), 2002 [doi: 10.2307/1558588].

Kerry D. Carson et Arthur G. Bedeian, « Career commitment: Construction of a measure and examination of its psychometric properties », Journal of Vocational Behavior, 44(3), 1994 [doi : 10.1006/jvbe.1994.1017].

Conference Board Canada, Bottom line: Labour shortages threaten tourism's growth - national summary, 2016 [http://emerit.ca/product/LMISUPDEM2.0-OL-E/en].

Wanda Costen et John Salazar, « The impact of training and development on employee job satisfaction, loyalty, and intent to stay in the lodging industry », Journal of Human Resources in Hospitality \& Tourism, 10(3), 2011 [doi : 10.1080/15332845.2011.555734].

W. R. Darden, R. Hampton et Roy D. Howell, « Career versus organizational commitment: Antecedents and consequences of retail salespeople's commitment », Journal of Retailing, 65(1), 1989.

Satya Dash, Edward Bruning et Kalyan Kumar Guin, « The moderating effect of power distance on perceived interdependence and relationship quality in commercial banking ", International Journal of Bank Marketing, 24(5), 2006 [doi : 10.1108/02652320610681747].

Maria T. M. Dijkstra, Bianca Beersma et Arne Evers, « Reducing conflict-related employee strain: The benefits of an internal locus of control and a problem-solving conflict management strategy », Work \& Stress, 25(2), 2011 [doi : 10.1080/02678373.2011.593344].

Thorsten Hennig-Thurau, « Customer orientation of service employees: Its impact on customer satisfaction, commitment, and retention ", International Journal of Service Industry Management, 15(5), 2004 [doi : 10.1108/09564230410564939].

Geert Hofstede, Gert Jan Hofstede et Michael Minkov, Cultures and organizations software of the mind: Intercultural cooperation and its importance for survival, MTM, 2017.

C. Harry Hui, Candice Yee et Karen L. Eastman, « The relationship between individualism collectivism and job satisfaction », Applied Psychology, 44(3), 1995 [doi : 10.1111/j. 1464-0597.1995.tb01080.x].

Nick Johns, Judy Henwood et Claire Seaman, « Culture and service predisposition among hospitality students in Switzerland and Scotland », International Journal of Contemporary Hospitality Management, $n^{\circ}$ 19, 2007 [doi : 10.1108/0959611071072956]. 
Hazel-Anne M. Johnson et Paul E. Spector, « Service with a smile: Do emotional intelligence, gender, and autonomy moderate the emotional labor process? ", Journal of Occupational Health Psychology, 12(4), 2007 [doi : 10.1037/1076-8998.12.4.319].

Peter Jones, Handbook of hospitality operations and IT, Butterworth Heinemann, 2008.

Juhee Kang et Sunghyup Sean Hyun, « Effective communication styles for the customer-oriented service employee: Inducing dedicational behaviors in luxury restaurant patrons », International Journal of Hospitality Management, 31(3), 2012 [doi: 10.1016/j.ijhm.2011.09.014].

Erdogan Koc, « Power distance and its implications for upward communication and empowerment: crisis management and recovery in hospitality services », The International Journal of Human Resource Management, 24(19), 2013 [doi : 10.1080/09585192.2013.778319].

Haiyan Kong et Xinyu Jiang, "Job satisfaction research in the field of hospitality and tourism ", International Journal of Contemporary Hospitality Management, 30(5), 2018 [doi : 10.1108/ IJCHM-09-2016-0525].

JungHoon Lee, Alleah Crawford, Melvin R. Weber et Dori Dennison, « Antecedents of cultural intelligence among american hospitality students: Moderating effect of ethnocentrism », Journal of Hospitality \& Tourism Education, 30(3), 2018 [doi : 10.1080/10963758.2018.1444494].

JungHoon Lee et Chihyung Ok, « Reducing burnout and enhancing job satisfaction: Critical role of hotel employees' emotional intelligence and emotional labor », International Journal of Hospitality Management, 31(4), 2012 [doi : 10.1016/j.ijhm.2012.01.007].

Darren Lee-Ross et Josephine Pryce, «A preliminary study of service predispositions amongst hospitality workers in Australia », The Journal of Management Development, 24(5/6), 2005 [doi : 10.1108/02621710510598436].

Manuel London et Raymond. A. Noe, «London's Career Motivation Theory: An Update on Measurement and Research », Journal of Career Assessment, 5(1), 1997 [doi : 10.1177/106907279700500105].

Arnaud Maurel, Magali Beffy et Denis Fougère, «L'impact du travail salarié des étudiants sur la réussite et la poursuite des études universitaires », Économie et Statistique, Insee, 2009.

Jum C. Nunnally, Psychometric theory, McGraw-Hill, 1978.

Barbara Plester et Ann Hutchison, « Fun times: The relationship between fun and workplace engagement », Employee Relations, 38(3), 2016 [doi : 10.1108/ER-03-2014-0027].

Julian B. Rotter, « Generalized expectancies for internal versus external control of reinforcement », Psychological Monographs: General and Applied, 80(1), 1966.

John Salazar, Susan Hubbard et Leta Salazar, « Locus of control and its influence on hotel managers' job satisfaction », Journal of Human Resources in Hospitality \& Tourism, 1(2), 2002 [doi : 10.1300/J171v01n02_02].

Diane Seymour, « Emotional labour: A comparison between fast food and traditional service work » International Journal of Hospitality Management, 19(2), 2000 [doi: 10.1016/ s0278-4319(00)00009-8].

Paula Silva, «Effects of disposition on hospitality employee job satisfaction and commitment ", International Journal of Contemporary Hospitality Management, 18(4), 2006 [doi : 10.1108/09596110610665320].

Steve Sizoo, Wilfried Iskat, Richard Plank et Hendrick Serrie, «Cross-Cultural Service Encounters in the Hospitality Industry and the Effect of Intercultural Sensitivity on Employee Performance », 
International Journal of Hospitality \& Tourism Administration, 4(2), 2004 [doi : 10.1300/

J149v04n02_04].

Marcia Taylor et Laura McArthur, « Cross-cultural knowledge, attitudes and experiences of hospitality management students », Journal of Hospitality \& Tourism Education, 21(4), 2009 [doi : 10.1080/10963758.2009.10696955].

Michael Tews et John Michel, « Workplace fun matters... but what else? », Employee Relations, 37(2), 2015 [doi : 10.1108/ER-10-2013-0152].

Jean M. Twenge, Liqing Zhang et Charles Im, « It's beyond my control: A cross-temporal metaanalysis of increasing externality in locus of control, 1960-2002 », Personality and Social Psychology Review, 8(3), 2004 [doi : 10.1207/s15327957pspr0803_5].

Christopher White, «The relationship between cultural values and individual work values in the hospitality industry », International Journal of Tourism Research, 7(4-5), 2005 [doi : 10.1002/jtr. 535].

Kathryn Frazer Winsted, "The service experience in two cultures: A behavioral perspective », Journal of Retailing, 73(3), 1997 [doi : 10.1016/S0022-4359(97)90022-1].

Boonghee Yoo, Naveen Donthu et Tomasz Lenartowicz, « Measuring Hofstede's five dimensions of cultural values at the individual level: Development and validation of CVSCALE », Journal of International Consumer Marketing, 23(3-4), 2011 [doi : 10.1080/08961530.2011.578059].

Choi Young Gin, Kwon Junehee et Kim Wansoo, « Effects of attitudes vs experience of workplace fun on employee behaviors: Focused on generation $Y$ in the hospitality industry », International Journal of Contemporary Hospitality Management, 25(3), 2013 [doi : 10.1108/09596111311311044].

\section{NOTES}

1. Le niveau collégial québécois offert dans les CÉGEP, correspond à un baccalauréat professionnel français.

\section{RÉSUMÉS}

$\mathrm{Au}$ cours de leur formation académique, les étudiants dans le domaine du tourisme, de l'hôtellerie et de la restauration ancrent lentement leurs choix de carrière dans l'industrie pour laquelle ils étudient. Cette recherche, basée sur la théorie des motivations de carrière de London et Noe (1997), établit un lien entre certaines dimensions intrinsèques des individus, telles que l'empreinte culturelle, les traits d'orientation client et le locus de contrôle, et leur appréciation des épisodes réels de travail au cours desquels des émotions positives sont vécues. Combinées aux prédispositions individuelles des étudiants, ces expériences concrètes déterminent la manière dont les étudiants s'engagent dans leur carrière naissante. Les résultats d'un sondage mené auprès de 424 étudiants du secteur du tourisme ont révélé qu'il existait une relation positive entre les étudiants ayant du plaisir au travail et la manière dont ils s'identifiaient, planifiaient et faisaient preuve de résilience face aux défis liés à leur carrière. 
During their academic training, students in the fields of tourism, hotel and restaurant management slowly anchor their career choices in the industry for which they study. This research, based on the theory of career motivations of London and Noe (1997), establishes a link between certain intrinsic dimensions of individuals, such as cultural imprint, customer orientation traits and locus of control, and their appreciation of the real work episodes during which positive emotions are experienced. Combined with the individual predispositions of the students, these concrete experiences determine the way in which students embark on their nascent career. Results of a survey of 424 tourism students revealed that there is a positive relationship between students who have fun at work and how they identify, plan and demonstrate resilience in the face of challenges related to their careers.

\section{INDEX}

Mots-clés : emploi, tourisme, carrières, modèle de motivation, étudiants

Keywords : employment, tourism, careers, motivation model, students

\section{AUTEURS}

\section{JEAN LAGUEUX}

Professeur, Université du Québec à Montréal

Gestion stratégique des entreprises touristiques

Lagueux.jean@uqam.ca

\section{AUDREY NANOT}

Professeure, Institut du tourisme et de l'hôtellerie du Québec Gestion de la diversité dans les entreprises touristiques audrey.nanot@ithq.qc.ca 